\title{
On the verbal width of finitely generated pro- $p$ groups
}

\section{Andrei Jaikin-Zapirain}

\begin{abstract}
Let $p$ be a prime. It is proved that a non-trivial word $w$ from a free group $F$ has finite width in every finitely generated pro- $p$ group if and only if $w \notin\left(F^{\prime}\right)^{p} F^{\prime \prime}$. Also it is shown that any word $w$ has finite width in a compact $p$-adic group.
\end{abstract}

\section{Introduction}

Let $F$ be a free group on $k$ independent generators. We will call an element $w$ from $F$ a word. If $G$ is a group, then we say that $g \in G$ is a $w$-value in $G$ if there are $g_{1}, \ldots, g_{k} \in G$ such that $g=w\left(g_{1}, \ldots, g_{k}\right)^{ \pm 1}$. We denote the set of the all $w$-values in $G$ by $G^{\{w\}}$. A simple argument (see [6]) shows that if $G$ is profinite, then $w(G)$ (the abstract subgroup generated by $G^{\{w\}}$ ) is closed if and only if there exists $l$ such that any element from $w(G)$ is a product of at most $l$ elements from $G^{\{w\}}$. The smallest such number $l$ is called the width of $w$ in $G$.

In this paper we consider a particular case of the following question: which words do have finite width in a finitely generated profinite group $G$ ? The most important achievement in this subject is a recent work of N. Nikolov and D. Segal (see [13]), where they proved that if $w$ is either $d$-locally finite or $w$ is a simple commutator, then $w$ has finite width in any $d$-generated profinite group $G$ (we recall that a group word $w$ is $d$-locally finite if every $d$-generator group $H$ satisfying $w(H)=1$ is finite).

The main result of this paper is as follows.

Theorem 1.1. Let $w \neq 1$ be an element of a free group $F$. Then the following are equivalent:

1. $w(H)$ is closed for every finitely generated pro-p group $H$;

2. $w \notin\left(F^{\prime}\right)^{p} F^{\prime \prime}$.

2000 Mathematics Subject Classification: Primary: 20E18; Secondary: 22E35.

Keywords: pro- $p$ group, verbal subgroup, verbal width, $p$-adic analytic group. 
The proofs of two implications in Theorem 1.1 are quite different. In order to prove $2 \Rightarrow 1$ we characterize the words $w \in F$ satisfying $w \notin$ $\left(F^{\prime}\right)^{p} F^{\prime \prime}$. We say that $w$ is a $\mathcal{N}_{p}$-word if for every finitely generated pro- $p$ group $H, H \overline{w(H)}$ is nilpotent-by-finite, where $\overline{w(H)}$ denotes the closure of

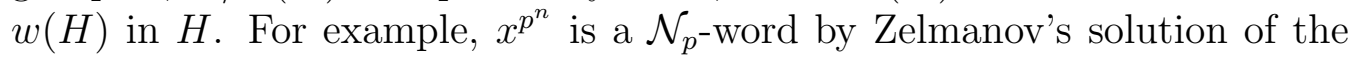
restricted Burnside problem. An Engel word is another example of $\mathcal{N}_{p}$-word (see [21]).

Theorem 1.2. Let $w$ be an element of a free group $F$. Then the following are equivalent:

1. $w$ is a $\mathcal{N}_{p}$-word;

2. if $H$ is a free pro-p group on two generators then $H / w(H)$ is nilpotentby-finite;

3. $w\left(C_{p} \prec \mathbb{Z}\right) \neq\{1\}$;

4. $w \notin\left(F^{\prime}\right)^{p} F^{\prime \prime}$.

This theorem reduces the proof of the implication $2 \Rightarrow 1$ from Theorem 1.1 to the case when $H$ is virtually nilpotent. This case is solved using the following more general result, which also answers a question posed by L. Pyber:

Theorem 1.3. Let $G$ be a compact $p$-adic analytic group. Then any word $w$ of a free group $F$ has finite width in $G$.

Note that in Theorem 1.3 we do not assume that $G$ is a pro- $p$ group.

In order to prove other implication from Theorem 1.1 we show that if $w \in\left(F^{\prime}\right)^{p} F^{\prime \prime}$ and $H$ is a non-abelian free pro- $p$ group, then $H^{\{w\}}$ is "very small" (more concretely we show that no power of $H^{\{w\}}$ can contain a nontrivial normal subgroup of $H$ ).

We use the following notation. If $S$ is a set and $m$ is a natural number then $S^{(m)}$ denotes the cartesian product of $m$ copies of $S$. If $S$ is a subset of a group $H$, then $S^{* m}$ is the set of all products $s_{1}^{ \pm 1} \cdots s_{n}^{ \pm 1}$, where $n \leq m$ and $s_{i} \in S$. We will also use the same notation when the operation in $H$ is additive, so in this case $S^{* m}=\left\{ \pm s_{1} \pm \cdots \pm s_{n} \mid n \leq m, s_{i} \in S\right\}$. We will say that $S$ has finite width in $H$ if there exists $l$ such that the subgroup $\langle S\rangle$ generated by $S$ is equal to $S^{* l}$. We use $[,]_{L}$ to denote the Lie bracket and simply [, ] for the group commutator.

Acknowledgements. I began work on Theorem 1.1 in collaboration with Pavel Shumyatsky, and thank him for kindly urging me to publish this contribution under my name, as well as for helpful conversations. Also I would like to thank Laci Pyber and Dan Segal for useful comments on preliminary versions of this paper. 


\section{Proof of Theorem 1.3}

In the following ||$_{p}$ is the standard $p$-adic valuation on $\mathbb{Q}_{p}$ : if $a \in p^{k} \mathbb{Z}_{p} \mid$ $p^{k+1} \mathbb{Z}_{p}$, then $|a|_{p}=p^{-k}$. Let $X=\left(X_{1}, \ldots, X_{m}\right)$ be $m$ commuting indeterminates and let $\mathbb{Q}_{p}[[X]]$ denote the set of formal power series over $\mathbb{Q}_{p}$. The ring $\mathbb{Q}_{p}\{X\}$ of restricted power series in $X$ over the $p$-adic field $\mathbb{Q}_{p}$ consists of the formal power series $\sum_{i} a_{i} X^{i}$ in $\mathbb{Q}_{p}[[X]]$ such that $\left|a_{i}\right|_{p} \rightarrow 0$ as $|i| \rightarrow \infty$. Here $i=\left(i_{1}, \ldots, i_{m}\right)$ ranges over $\mathbb{Z}_{\geq 0}^{(m)},|i|=i_{1}+\cdots+i_{m}, X^{i}=X_{1}^{i_{1}} \cdots X_{m}^{i_{m}}$. Let $f=\sum_{i} a_{i} X^{i}$ be in $\mathbb{Q}_{p}\{X\}$ and $x \in \mathbb{Z}_{p}^{(m)}$. Then the series $\sum_{i} a_{i} x^{i}$ converges to a limit in $\mathbb{Q}_{p}$ which we denote by $f(x)$. The subring $\mathbb{Z}_{p}\{X\}$ of $\mathbb{Q}_{p}\{X\}$ consists of the series $\sum_{i} a_{i} X^{i}$ in $\mathbb{Q}_{p}\{X\}$ all of whose coefficients are in $\mathbb{Z}_{p}$.

Let $L=\left(\mathbb{Z}_{p}^{(m)},+\right)$. We will consider $L$ as a $p$-adic manifold. Hence for each points $a$ of $L$ we can define a local ring $H_{a}$ at $a$ with the maximal ideal $\mathfrak{m}_{a}$. The dual of $\mathfrak{m}_{a} / \mathfrak{m}_{a}^{2}$ is the tangent space of $L$ at $a$ and it is denoted by $T_{a} L$. If $K$ is another $p$-adic manifold and $g: K \rightarrow L$ is an analytic map, then the induced map of the tangent spaces $T_{x} K \rightarrow T_{y} L(y=g(x))$ is denoted by $T_{x} g$. For details, see [18, Part II. Chapter III]. We denote the element $(0, \ldots, 0)$ by $\mathbf{e}$.

Lemma 2.1. Let $Y=\left(Y_{1}, \ldots, Y_{n}\right)$ and $f=\left(f_{1}, \ldots, f_{m}\right)$ be a m-tuple consisting of $m$ formal power series from $\mathbb{Z}_{p}\{Y\}$ such that $f(\boldsymbol{e})=\boldsymbol{e}$. Put

$$
S=f\left(\mathbb{Z}_{p}^{(n)}\right)=\left\{\left(f_{1}(x), \ldots, f_{m}(x)\right) \mid x \in \mathbb{Z}_{p}^{(n)}\right\} \subseteq \mathbb{Z}_{p}^{(m)}
$$

Then the width of $S$ in $\left(\mathbb{Z}_{p}^{(m)},+\right)$ is finite.

Proof. Put $K=\mathbb{Z}_{p}^{(n)}$ and $L=\left(\mathbb{Z}_{p}^{(m)},+\right)$ and let $A$ be the closed subgroup of $L$ generated by $S$. If $L_{1}=\left\{l \in L \mid p^{k} l \in A\right.$ for some $\left.k\right\}$, then we can find a subgroup $L_{2}$ of $L$ such that $L=L_{1} \oplus L_{2}$. We can choose new coordinates $\left\{x_{1}, \ldots, x_{m}\right\}$ of $L$ such that $L_{1}$ is given by equations $\left\{x_{s+1}=\cdots=x_{m}=0\right\}$. Then in new coordinates the map $f$ looks like $\left(h_{1}, \ldots, h_{s}, 0, \ldots, 0\right)$. Hence, without loss of generality, we can suppose that $S$ generates an open subgroup in $L$. We may clearly assume that $L \neq 0$.

For any $a \in K$ define $g_{a}(Y)=f(Y)-f(a)$. Then $g_{a}$ is an analytic map from $K$ to $L$ and $g_{a}(a)=\mathbf{e}$. This analytic map induces a map between the tangent space $T_{a} g_{a}: T_{a} K \rightarrow T_{\mathbf{e}} L$. Let us calculate the image of this map.

Let $\partial_{i}$ be the partial derivation with respect to the $i$ th coordinate. Then the functionals $e_{i}: \mathfrak{m}_{a} / \mathfrak{m}_{a}^{2} \rightarrow \mathbb{Q}_{p}$ defined by $e_{i}(q)=\left(\partial_{i} q\right)(a)$ form a basis of $T_{a} K$. In the same way the functionals $h_{i}: \mathfrak{m}_{\mathbf{e}} / \mathfrak{m}_{\mathbf{e}}^{2} \rightarrow \mathbb{Q}_{p}$ defined by $h_{i}(q)=\left(\partial_{i} q\right)(\mathbf{e})$ form a basis of $T_{\mathbf{e}} L$. 
Notice that $\left(T_{a} g_{a}\right)\left(e_{i}\right)\left(x_{j}\right)=e_{i}\left(f_{j}-f_{j}(a)\right)=\partial_{i} f_{j}(a)$. Thus,

$$
\left(T_{a} g_{a}\right)\left(e_{i}\right)=\sum_{j=1}^{m} \partial_{i} f_{j}(a) h_{j} .
$$

Now consider the subspace of $T_{\mathbf{e}} L$ generated by all images of $T_{a} g_{a}$ for all $a$. If this subspace is different from $T_{\mathbf{e}} L$, then there are constants $\alpha_{1}, \ldots, \alpha_{m}$, not all zero, such that for all $a \in K$ and $1 \leq i \leq N$,

$$
0=\sum_{j=1}^{m} \alpha_{j} \partial_{i} f_{j}(a)=\partial_{i}\left(\sum_{j=1}^{m} \alpha_{j} f_{j}\right)(a) .
$$

Thus $\partial_{i}\left(\sum_{j=1}^{m} \alpha_{j} f_{j}\right)=0$ for all $i$. Hence $g=\sum_{j=1}^{m} \alpha_{j} f_{j}$ is a constant function. But since $g(\mathbf{e})=\mathbf{e}, g$ is the zero function. But this contradicts the assumption that $S$ generates an open subgroup in $L$.

Hence the subspace of $T_{\mathbf{e}} L$ generated by all images of $T_{a} g_{a}$ is equal to $T_{\mathbf{e}} L$. Therefore we can find $m$ elements $a_{1}, \ldots a_{m}$ in $K$ such that

$$
T_{a_{1}} g_{a_{1}}\left(T_{a_{1}} K\right)+\cdots+T_{a_{m}} g_{a_{m}}\left(T_{a_{m}} K\right)=T_{\mathbf{e}} L
$$

Define a map $h$ from $K^{(m)}$ to $L$ by $h\left(b_{1}, \ldots, b_{m}\right)=g_{a_{1}}\left(b_{1}\right)+\cdots+g_{a_{m}}\left(b_{m}\right)$. Put $b=\left(a_{1}, \ldots, a_{m}\right)$. Then, by $2.1, T_{b} h\left(T_{b} K^{(m)}\right)=T_{\mathbf{e}} L$. Hence from [18, Theorem 10.2, p.85] we obtain that $h$ is a submersion and so $h\left(K^{(m)}\right)$ contains an open in $L$ subset. Thus $S^{* m}$ contains an open in $A=\langle S\rangle$ subset.

Since $A$ is a profinite group, there exists an open subgroup $B$ of $A$ and $a \in$ $A$ such that $a+B \subseteq S^{* m}$. Since $A=S^{* l}+B$ for some $l, A=S^{*(m+l)}$.

Recall that a pro- $p$ group $G$ is called powerful if $[G, G] \leq G^{p}$ when $p>2$ or $[G, G] \leq G^{4}$ when $p=2$. We say that a finitely generated pro- $p$ group $G$ is uniform if $G$ is powerful and without torsion. A uniform $\mathbb{Z}_{p^{-}}$ Lie lattice is a Lie ring $L$ such that $L$ is a finitely generated free $\mathbb{Z}_{p}$-module and $[L, L]_{L} \leq p L$ when $p>2$ or $[L, L]_{L} \leq 4 L$ when $p=2$. According to Lazard (see, for example, [4]), there is an equivalence between the category of uniform pro- $p$ groups and the category of uniform $\mathbb{Z}_{p}$-Lie lattices. The uniform $\mathbb{Z}_{p}$-Lie lattice $\mathrm{H}$ corresponding to a uniform pro- $p$ group $H$ has $H$ itself as its underlying set and the Lie ring operations are given in terms of the group operations as follows: for all $z \in \mathbb{Z}_{p}$ and all $x, y \in H$ we have

$$
\begin{aligned}
z \cdot x & =x^{z}, \\
g+h & =\lim _{n \rightarrow \infty}\left(g^{p^{n}} h^{p^{n}}\right)^{p^{-n}}, \\
{[g, h]_{L} } & =\lim _{n \rightarrow \infty}\left[g^{p^{n}}, h^{p^{n}}\right]^{p^{-2 n}} .
\end{aligned}
$$


Conversely, given a uniform $\mathbb{Z}_{p}$-Lie lattice $\mathrm{H}$ of rank $m$, the uniform pro- $p$ group $H$ corresponding to $\mathrm{H}$ can be constructed via the Baker-CampbellHausdorff formula. Its underlying set is again $\mathrm{H}$, and the group product of $x, y \in \mathrm{H}$ is given by $x y=\Phi(x, y)$. Recall that the Baker-Campbell-Hausdorff formula is $\Phi\left(x_{1}, x_{2}\right)=\log \left(e^{x_{1}} e^{x_{2}}\right)$ regarded as a formal power series in two non-commuting variables (see [4, Section II.6.5]).

From now on we fix a system of free $\mathbb{Z}_{p}$-generators of $\mathrm{H}$. Thus to any element $x$ from $\mathrm{H}$ corresponds a $m$-tuple $\left(x_{1}, \ldots, x_{m}\right)$ from $\mathbb{Z}_{p}^{(m)}$. We will refer to this $m$-tuple as coordinates of $x$ and we will regard $\mathrm{H}$ (and so $H$ ) as $\mathbb{Z}_{p}^{(m)}$. Then, by [2, Proposition II.8.1]), the multiplication in $H$ is given by a $m$-tuple $\left(F_{1}, \ldots, F_{m}\right)$ where $F_{i} \in \mathbb{Z}_{p}\{X\}$.

Corollary 2.2. Let $Y=\left(Y_{1}, \ldots, Y_{n}\right)$ and $f=\left(f_{1}, \ldots, f_{m}\right)$ be a m-tuple consisting of $m$ formal power series from $\mathbb{Z}_{p}\{Y\}$. Put

$$
S=f\left(\mathbb{Z}_{p}^{(n)}\right)=\left\{\left(f_{1}(x), \ldots, f_{m}(x)\right) \mid x \in \mathbb{Z}_{p}^{(n)}\right\} \subseteq H .
$$

Suppose that $f(\boldsymbol{e})=\boldsymbol{e}$ and the group generated by $S$ is abelian. Then the width of $S$ in $H$ is finite.

Proof. Since the group $\langle S\rangle$ is abelian, we can apply Lemma 2.1, because the width of $S$ in $H$ is the same as the width of $S$ in $(\mathrm{H},+)$.

Lemma 2.3. Let $Y=\left(Y_{1}, \ldots, Y_{n}\right)$ and $f=\left(f_{1}, \ldots, f_{m}\right)$ be a m-tuple consisting of $m$ formal power series from $\mathbb{Z}_{p}\{Y\}$. Put

$$
S=f\left(\mathbb{Z}_{p}^{(n)}\right)=\left\{\left(f_{1}(x), \ldots, f_{m}(x)\right) \mid x \in \mathbb{Z}_{p}^{(n)}\right\} \subseteq H .
$$

Suppose that $f(\boldsymbol{e})=\boldsymbol{e}$ and $S$ is a normal set in $H$. Then the width of $S$ in $H$ is finite.

Proof. Let $T$ be the closed subgroup generated by $S$. Since $S$ is a normal set in $H, T$ is a normal subgroup of $H$. Hence the set

$$
R=\left\{x \in H \mid x^{p^{k}} \in[T, T] \text { for some } k\right\}
$$

is also normal subgroup in $H$. Put $\bar{H}=H / R$. Note that $\bar{H}$ is a uniform pro- $p$ group. Moreover, $R$ is an ideal of $\mathrm{H}$. Hence we can choose new coordinates $\left\{x_{1}, \ldots, x_{m}\right\}$ of $\mathrm{H}$ in such way that $R$ is defined by equations $\left\{x_{1}=\cdots=x_{s}=0\right\}$. Suppose that in these new coordinates the map $f$ looks like $\left(g_{1}, \ldots, g_{m}\right)$. Note that the first $s$ coordinates $\left(x_{1}, \ldots, x_{s}\right)$ of $\mathrm{H}$ determine uniquely an element $\bar{x}$ from $\overline{\mathrm{H}}$ and they are coordinates of $\bar{x}$ with respect to some system of $\mathbb{Z}_{p}$-generators of $\bar{H}$. Thus, the composition of $f$ with the natural epimorphism to $\overline{\mathrm{H}}$ looks like $\left(g_{1}, \ldots, g_{s}\right)$. Since the set $\bar{S}=S R / R$ 
generates an abelian subgroup in $\bar{H}$, we obtain from the previous corollary that there exists $l_{1}$ such that $T=S^{* l_{1}} R$. On the other hand $R /[T, T]$ is finite, whence we also obtain that $T=S^{* l_{2}}[T, T]$ for some $l_{2}$.

Since $H$ is uniform, $T$ is finitely generated. Let $t_{1}, \ldots, t_{l} \in S$ be the generators of $T$ as a pro- $p$ group. Then $[T, T]=\left[t_{1}, T\right] \cdots\left[t_{l}, T\right]$ (see $[4$, Proof of Proposition 1.19]) and so, since $S$ is normal, $[T, T] \subseteq S^{* 2 l}$. Hence $T=S^{*\left(2 l+l_{2}\right)}$.

Proof of Theorem 1.3. Since $G$ is $p$-adic analytic, $G$ has an open uniform normal pro- $p$ subgroup $H$ (see [4, Corollary 8.34]). Let $\left\{a_{i}|1 \leq i \leq| G: H \mid\right\}$ be a tranversal of $G$ over $H$. For each $i=\left(i_{1}, \ldots, i_{k}\right)$ define the function $g_{i}: H^{(2 k)} \rightarrow H$ by means of

$$
g_{i}\left(h_{1, i}, \ldots, h_{2 k, i}\right)=w\left(a_{i_{1}} h_{1, i}, \ldots, a_{i_{k}} h_{k, i}\right) w\left(a_{i_{1}} h_{k+1, i}, \ldots, a_{i_{k}} h_{2 k, i}\right)^{-1} .
$$

Choose any order on $k$-tuples and put $f=\prod_{i} g_{i}$. Then $f$ is a function from $H^{2 k|G: H|^{k}}$ to $H$. Moreover, if we regard $H$ as $\mathbb{Z}_{p}^{(m)}$ then $f$ is an $m$-tuple of functions from $\mathbb{Z}_{p}\{Y\}$, where $Y=\left(Y_{1}, \ldots, Y_{n}\right)$ and $N=2 M k|G: H|^{k}$. Put $S=f\left(\mathbb{Z}_{p}^{(n)}\right)$ and let $T$ be the closed subgroup generated by $S$.

If $h, h_{1}, \ldots, h_{k} \in H$ and $1 \leq i_{1}, \ldots, i_{k} \leq|G: H|$, then

$$
w\left(a_{i_{1}} h_{1}, \ldots, a_{i_{k}} h_{k}\right)^{h}=w\left(a_{i_{1}}\left[a_{i_{1}}, h\right] h_{1}^{h}, \ldots, a_{i_{k}}\left[a_{i_{k}}, h\right] h_{k}^{h}\right) .
$$

Therefore $S$ is a normal set in $H$, and so, $T$ is a normal subgroup of $G$. By the previous lemma, there exists $l$ such that $T=S^{* l}$. Since $S^{* l} \subseteq$ $\left(G^{\{w\}}\right)^{* 2 l M k|G: H|^{k}}$, we obtain that $T \subseteq\left(G^{\{w\}}\right)^{* m_{1}}$ for some $m_{1}$.

Consider the group $\bar{G}=G / T$. Note that the word $w$ takes only finitely many different values in $\bar{G}$. Since $\bar{G}$ is a $p$-adic analytic group, it is linear. Hence, by Merzlyakov's solution of Hall's problem for linear groups [10], $w(\bar{G})=w(G) / T$ is finite. Thus, $w(G)=\left(G^{\{w\}}\right)^{* m_{2}} T=\left(G^{\{w\}}\right)^{*\left(m_{1}+m_{2}\right)}$ for some $m_{2}$.

We recall that P. Hall's question is

Question. Let $G$ be a group and $w$ a word from a free group. Suppose that the word $w$ takes only finitely many different values in $G$. Is it true that $w(G)$ is finite?

S. Ivanov [8] answered this question for arbitrary groups in the negative; he constructed a group $H$ and a word $w(x, y)$ such that $w(H)$ is infinite cyclic but $w(x, y)$ has only one non-trivial value in $H$. Ivanov's example is not residually finite. So as far as I know, P. Hall's question for profinite groups is still open. 


\section{Verbal subgroups corresponding to $\mathcal{N}_{p}$-words}

In this section we prove that if $H$ is a finitely generated pro- $p$ group then $w(H)$ is closed for any $\mathcal{N}_{p}$-word $w$. First we prove Theorem 1.2.

Proof of Theorem 1.2. The implications $1 \Rightarrow 2 \Rightarrow 3 \Rightarrow 4$ are clear.

Suppose now that the third condition holds. If $w$ is not a $\mathcal{N}_{p}$-word, then by [3] (see also [17]), there is a finitely generated non $p$-adic analytic pro- $p$ group $H$ such that $w(H)=1$. From [19] it follows that $w\left(C_{p} \prec \mathbb{Z}\right)=1$, a contradiction. Hence $3 \Rightarrow 1$.

Now $4 \Rightarrow 3$ follows, for example, from [11, Theorem 22.43].

Theorem 3.1. Let $w$ be a $\mathcal{N}_{p}$-word and $G$ a finitely generated pro-p group. Then $w(G)$ is closed.

Proof. Let $d=d(G)$ and $H$ be a free pro- $p$ group on generators $x_{1}, \ldots, x_{d}, z$. Since $w$ is a $\mathcal{N}_{p^{-w o r d}} \gamma_{n}\left(H^{p^{t}}\right) \leq \overline{w(H)}$ for some $n$ and $t$.

Denote by $y_{1}, \ldots, y_{s}$ generators of $\overline{\left\langle x_{1}, \ldots, x_{d}\right\rangle^{t}}$. Note that $y_{i}$ are pro- $p$ words in $x_{i}$ (and do not involve $z$ ). By Theorem 1.3, there exists $k$ such that for any, $i_{1}, \ldots, i_{n} \in\{1, \ldots, s\}$,

$$
\left[z, y_{i_{1}}, y_{i_{2}}, \ldots, y_{i_{n}}\right] \equiv v_{i_{1}, \ldots, i_{n}} \quad\left(\bmod \gamma_{n+2}\left(H^{p^{t}}\right)\right),
$$

where $v_{i_{1}, \ldots, i_{n}}$ is a product of at most $k w$-values in $H$. Thus,

$$
v_{i_{1}, \ldots, i_{n}}\left(x_{1}, \ldots, x_{d}, z\right)=\left[z, y_{i_{1}}, y_{i_{2}}, \ldots, y_{i_{n}}\right] r_{i_{1}, \ldots, i_{n}}\left(x_{1}, \ldots, x_{d}, z\right)
$$

with $r_{i_{1}, \ldots, i_{n}}\left(x_{1}, \ldots, x_{d}, z\right) \in \gamma_{n+2}\left(H^{p^{t}}\right)$.

Let $h_{1}, \ldots, h_{d}$ be generators of $G$.

Claim 1. Let $a \in \gamma_{m}\left(G^{p^{t}}\right), g \in G$ and $r \in \gamma_{l}\left(H^{p^{t}}\right)$. Then

$$
r\left(h_{1}, \ldots, h_{d}, g\right) \equiv r\left(h_{1}, \ldots, h_{d}, g a\right) \quad\left(\bmod \gamma_{m+l-1}\left(G^{p^{t}}\right)\right) .
$$

When $l=1$, the claim is clear. The general case follows by induction on $l$.

Claim 2. $\gamma_{n+1}\left(G^{p^{t}}\right)=\prod_{i_{1}, \ldots, i_{n}} v_{i_{1}, \ldots, i_{n}}\left(h_{1}, \ldots, h_{d}, G^{p^{t}}\right)$.

By induction on $m$ we prove that if $m \geq n+1$ then

$$
\gamma_{n+1}\left(G^{p^{t}}\right)=\prod_{i_{1}, \ldots, i_{n}} v_{i_{1}, \ldots, i_{n}}\left(h_{1}, \ldots, h_{d}, H^{p^{t}}\right) \gamma_{m}\left(G^{p^{t}}\right) .
$$

This implies the claim because the set

$$
\prod_{i_{1}, \ldots, i_{n}} v_{i_{1}, \ldots, i_{n}}\left(h_{1}, \ldots, h_{d}, H^{p^{t}}\right)
$$

is closed. 
The base of induction $m=n+1$ is clear. Suppose it holds for $m$. Let prove it for $m+1$. Let $h \in \gamma_{n+1}\left(G^{p^{t}}\right)$. By the inductive hypothesis, there are $g_{i_{1}, \ldots, i_{n}} \in H^{p^{t}}$ and $u \in \gamma_{m}\left(G^{p^{t}}\right)$ such that

$$
h=\prod_{i_{1}, \ldots, i_{n}} v_{i_{1}, \ldots, i_{n}}\left(h_{1}, \ldots, h_{d}, g_{i_{1}, \ldots, i_{n}}\right) u
$$

We can write

$$
u \equiv \prod_{i_{1}, \ldots, i_{n}}\left[t_{i_{1}, \ldots, i_{n}}, \tilde{y}_{i_{1}}, \ldots, \tilde{y}_{i_{n}}\right] \quad\left(\bmod \gamma_{m+1}\left(G^{p^{t}}\right)\right)
$$

where $\tilde{y}_{j}=y_{j}\left(h_{1}, \ldots, h_{d}\right)$ and $t_{i_{1}, \ldots, i_{n}} \in \gamma_{m-n}\left(G^{p^{t}}\right)$. Thus,

$$
\begin{array}{r}
h \equiv \prod_{i_{1}, \ldots, i_{n}}\left[g_{i_{1}, \ldots, i_{n}} t_{i_{1}, \ldots, i_{n}}, \tilde{y}_{i_{1}}, \ldots, \tilde{y}_{i_{n}}\right] r_{i_{1}, \ldots, i_{n}}\left(h_{1}, \ldots, h_{d}, g_{i_{1}, \ldots, i_{n}}\right) \\
\left(\bmod \gamma_{m+1}\left(G^{p^{t}}\right)\right) .
\end{array}
$$

By Claim 1,

$$
\begin{array}{r}
r_{i_{1}, \ldots, i_{n}}\left(h_{1}, \ldots, h_{d}, g_{i_{1}, \ldots, i_{n}}\right) \equiv r_{i_{1}, \ldots, i_{n}}\left(h_{1}, \ldots, h_{d}, g_{i_{1}, \ldots, i_{n}} t_{i_{1}, \ldots, i_{n}}\right) \\
\left(\bmod \gamma_{m+1}\left(G^{p^{t}}\right)\right) .
\end{array}
$$

Hence we have

$$
h \equiv \prod_{i_{1}, \ldots, i_{n}} v_{i_{1}, \ldots, i_{n}}\left(h_{1}, \ldots, h_{d}, g_{i_{1}, \ldots, i_{n}} t_{i_{1}, \ldots, i_{n}}\right) \quad\left(\bmod \gamma_{m+1}\left(G^{p^{t}}\right)\right) .
$$

This finishes the proof of the claim.

It follows that the closed subgroup $\gamma_{n+1}\left(G^{p^{t}}\right)$ is contained in $w(G)$. We may therefore apply Theorem 1.3 and deduce that

$$
w(G) / \gamma_{n+1}\left(G^{p^{t}}\right)=w\left(G / \gamma_{n+1}\left(G^{p^{t}}\right)\right.
$$

is closed. Hence $w(G)$ is closed.

\section{Words of infinite width}

In this section we prove that if $F$ is a non-abelian free group and $1 \neq w \in$ $\left(F^{\prime}\right)^{p} F^{\prime \prime}$, then $w(H)$ is not closed in a free finitely generated non-abelian free pro- $p$ group $H$. These examples generalize the example of Roman'kov [15] who proved the same statement for $w=[[x, y],[z, u]]$.

Theorem 4.1. Let $F$ be a non-abelian free group, $p$ a prime number and $H$ a non-abelian finitely generated free pro-p group. Suppose that $1 \neq w \in$ $\left(F^{\prime}\right)^{p} F^{\prime \prime}$. Then the verbal subgroup $w(H)$ is not closed. 
We explain first the strategy of the proof of this theorem. We suppose the contrary, that there exists $k$ such that any element of $w(H)$ is a product of at most $k w$-values in $H$. Hence since $w \in\left(F^{\prime}\right)^{p} F^{\prime \prime}$ there exists a number $l$ depending on $w$ such that any element of $w(H)$ is a product of at most $l$ values of the word $x^{p}[y, z]$ in $H^{\prime}$. Now, note that $H^{\prime}$ is a free pro- $p$ group of infinite rank and $w(H)$ is a normal subgroup of $H^{\prime}$. Then the following proposition leads us to a contradiction.

Proposition 4.2. 1. Let $K$ be a free pro-p group of rank $d$ and $\{1\} \neq N$ a closed normal subgroup of $K$. Then there exists an element $g \in N$ such that $g$ cannot be represented as a product of less than $[d / 3]$ values of the word $x^{p}[y, z]$ in $K$.

2. Let $K$ be a free pro-p group of infinite rank and $\{1\} \neq N$ a closed normal subgroup of $K$. Then for any $l \in \mathbb{N}$ there exists an element $g \in N$ such that $g$ cannot be represented as a product of less than $l$ values of the word $x^{p}[y, z]$ in $K$.

Before the proof of the proposition we present an auxiliary result. For any pro- $p$ group $G$, let $D_{i}(G)$ be the $i$ th dimension subgroup of $G$ (see e.g. [4, Chapter 11]). Let $K$ be a free pro- $p$ group of rank $d$ and $\{1\} \neq N$ a closed normal subgroup of $K$. Put $N_{i}=N \cap D_{i}(K)$ and define the following numbers:

$$
\begin{aligned}
& a_{i}=\log _{p}\left|D_{i}(K): D_{i+1}(K)\right|, \quad b_{i}=\log _{p}\left|K: D_{i+1}(K)\right|, \\
& c_{i}=\log _{p}\left|N_{i}: N_{i+1}\right|, \quad d_{i}=\log _{p}\left|N: N_{i+1}\right|=\log _{p}\left|N D_{i+1}(K): D_{i+1}(K)\right| .
\end{aligned}
$$

Lemma 4.3. When $n$ tends to infinity the following holds

1. $a_{n}=\frac{d^{n}}{n}(1+o(1))$;

2. $b_{n}=\frac{d^{n+1}}{(d-1) n}(1+o(1))$;

3. $c_{n}=\frac{d^{n}}{n}(1+o(1))$;

4. $d_{n}=\frac{d^{n+1}}{(d-1) n}(1+o(1))$.

Proof. Let $L$ be a free Lie algebra generated by $d$ elements. Then $L$ can be graded in a standard way if we suppose that free generators are elements of degree 1 . Then we can write $L=\oplus_{i} L_{i}$. The following formula

$$
\operatorname{dim} L_{i}=M_{d}(n)=\frac{1}{n} \sum_{k \mid n} \mu(k) d^{n / k}
$$

is well-known. Moreover, $M_{d}(n)=\frac{d^{n}}{n}(1+o(1))$ (see [7, Chapter VIII]). 
Recall that if $G$ is a pro- $p$ group, then $L_{p}(G)=\oplus D_{i}(G) / D_{i+1}(G)$ has the structure of a restricted Lie $\mathbb{F}_{p^{-}}$algebra. Moreover, $L_{p}(K)$ is a free $p$-restricted Lie $\mathbb{F}_{p^{-}}$algebra. The construction of a free Lie restricted $\mathbb{F}_{p^{-}}$ algebra from a free Lie $\mathbb{F}_{p}$-algebra is described, for example, in $[2$, Exercise 2.3.4]. It follows that if $n=p^{s} m$, where $m$ and $p$ are coprime, then

$$
a_{n}=\log _{p}\left|D_{n}(K): D_{n+1}(K)\right|=\sum_{k=0}^{s} M_{d}\left(m p^{k}\right) .
$$

This is an easy exercise to obtain from this that $a_{n}=\frac{d^{n}}{n}(1+o(1))$.

Now, since

$$
\lim _{n \rightarrow \infty} a_{n}=\lim _{n \rightarrow \infty} b_{n}=\infty \text { and } \lim _{n \rightarrow \infty} \frac{a_{n+1}-a_{n}}{b_{n+1}-b_{n}}=\frac{d-1}{d},
$$

we obtain that $\lim _{n \rightarrow \infty} \frac{a_{n}}{b_{n}}=\frac{d-1}{d}$. Hence $b_{n}=\frac{d^{n+1}}{(d-1) n}(1+o(1))$.

Put $G=K / N$. Then $p$-enveloping algebra of $L_{p}(G)$ is a proper quotient of a free $\mathbb{F}_{p}$-algebra on $d$-generators. Hence, by [5] (see also [12, Theorem 3.1] and [14, Theorem 15]), there exists $\alpha<d$ such that

$$
a_{i}-c_{i}=\log _{p}\left|D_{i}(G) / D_{i+1}(G)\right| \leq \alpha^{n}
$$

when $n$ tends to infinity. This inequality with the previous estimation for $a_{n}$ implies that $c_{n}=\frac{d^{n}}{n}(1+o(1))$. As in the case $b_{n}$, we obtain that $d_{n}=$ $\frac{d^{n+1}}{(d-1) n}(1+o(1))$.

Now we are ready to prove Proposition 4.2.

Proof of Proposition 4.2. 1. Put $V_{n}=\left\{g_{1}^{p}\left[g_{2}, g_{3}\right] \in K / D_{n+1}(K) \mid g_{i} \in\right.$ $\left.K / D_{n+1}(K)\right\}$. Then, by Lemma 4.3 ,

$$
\left|V_{n}\right| \leq\left|K / D_{n}(K)\right|^{3}=p^{3 b_{n-1}}=p^{\frac{3 d^{n}}{(d-1)(n-1)}(1+o(1))} .
$$

On the other hand applying again Lemma 4.3, we obtain that

$$
\left|N D_{n+1}(K) / D_{n+1}(K)\right|=p^{\frac{d^{n+1}}{(d-1) n}(1+o(1))} .
$$

Comparing these two expressions we obtain 1.

2. In order to prove 2 , it is enough to observe that for any $d>1$, a free pro- $p$ group of infinite rank is residually-free pro- $p$ of rank $d$. Hence for any $d>1$ there exists a homomorphism of $K$ onto a free pro- $p$ group of rank $d$ such that the image of $N$ is not trivial. Then we can apply 1 . Since $d$ is arbitrary, we obtain 2 . 
Remark 4.4. The first statement of Proposition 4.2 has the following interpretation. For any closed subset $V$ of $K$ define its Hausdorff dimension (see [1]):

$$
\operatorname{dim}_{H} V=\liminf _{n \rightarrow \infty} \frac{\log \left|V D_{n}(K): D_{n}(K)\right|}{\log \left|K: D_{n}(K)\right|} .
$$

In the proof of Proposition 4.2 we have shown that $\operatorname{dim}_{H} N=1$ for any nontrivial normal subgroup $N$ of $K$ and $\operatorname{dim}_{H} K^{\left\{x^{p}[y, z]\right\}} \leq \frac{3}{d}$.

\section{Final remarks}

\subsection{The finite verbal width and the Restricted Burnside Problem}

If $H$ is a finitely generated pro- $p$ group and $w=x^{p^{n}}$, then all known proofs that $w(H)$ is closed use the Zelmanov's solution of the Restricted Burnside Problem. For example, from Zelmanov's result it follows that $w$ is a $\mathcal{N}_{p}$-word and then we can apply Theorem 3.1. In [16, page 53], Dan Segal suggested that it would be very interesting to prove that $H^{p^{n}}$ is closed without appealing to Zelmanov's result. Using the ideas of the previous section we will show that it would give an alternative solution of the Restricted Burnside Problem.

Theorem 5.1. Let $F$ be a free group and $w \in F$. Put $t=w^{p}$. Then if $t(G)$ is closed for any finitely generated pro-p group $G$, then $w(G)$ is open for any finitely generated pro-p group $G$.

Proof. Note that in the proof of Theorem 3.1 we have used the solution of the Restricted Burnside Problem (we needed $H^{p^{s}}$ to be open). However, if we assume that $w$ satisfies a stronger condition: $G / \overline{w(G)}$ is nilpotent for any finitely generated pro- $p$ group $G$, then we obtain that $w$ is of finite width without appealing to the solution of the Restricted Burnside Problem.

Let, now, $H$ be a non abelian finitely generated free pro- $p$ group and $\overline{w(H)}$ the closure of $w(H)$ in $H$. First we assume that $\overline{w(H)}$ is not open. Since $H$ is a non-abelian free pro- $p$ group, then $\overline{w(H)}$ is a free pro- $p$ group of infinite rank. Now, using the argument of the proof of Theorem 4.1, we obtain that $t(H)$ is not closed.

Hence we can assume that $\overline{w(H)}$ is open. In particular, $H \overline{w(H)}$ is nilpotent. Thus, from the first paragraph we obtain that $w(H)$ is also closed and so open.

\subsection{Pronilpotent groups}

In this subsection we show how our previous results on pro- $p$ groups can be generalized on pronilpotent groups. The possibility of this generalization has been suggested to us by Dan Segal. 
We say that $w$ is a $\mathcal{N}$-word if for any finitely generated pronilpotent group $H, H \overline{w(H)}$ is nilpotent-by-finite, where $\overline{w(H)}$ denotes the closure of $w(H)$ in $H$. The following characterization of $\mathcal{N}$-words is due to Dan Segal.

Theorem 5.2. Let $w$ be an element of a free group $F$. Then the following are equivalent

1. $w$ is a $\mathcal{N}$-word;

2. $w$ is a $\mathcal{N}_{p}$-word for all primes $p$.

Proof. The implication $1 \Rightarrow 2$ is clear. Let us prove $2 \Rightarrow 1$.

Let $d$ be a natural number, $U$ a free group on $d$ generators and $T$ the maximal residually nilpotent quotient of $U / w(U)$. Since $w$ is a $\mathcal{N}_{2}$-word, the pro- 2 completion $T_{\hat{2}}$ of $T$ is virtually nilpotent, and so of finite rank. Using [9, Lemma 9, page 386], we obtain that there exists a finite set $\pi$ of primes such that $T$ is embedded in $\prod_{p \in \pi} T_{\hat{p}}$. Applying again that $w$ is a $\mathcal{N}_{p}$-word for all primes $p \in \pi$, we conclude that $T$ is virtually nilpotent.

Let now $H$ be a finitely generated pronilpotent group and $\bar{H}=H \overline{w(H)}$. Put $d=d(H)$. Let $T_{1}$ be a dense $d$-generated subgroup of $\bar{H}$. Then, $T_{1}$ is a quotient of $T$. Hence $T_{1}$ and $\bar{H}$ are virtually nilpotent.

Now, we are ready to prove the main theorem of this subsection.

Theorem 5.3. Let $1 \neq w$ be an element of a free group $F$. Then the following two statements are equivalent:

1. $w(H)$ is closed for every finitely generated pronilpotent group $H$;

2. $w \notin \bigcup_{p \text { prime }}\left(F^{\prime}\right)^{p} F^{\prime \prime}$.

Proof. The implication $1 \Rightarrow 2$ follows from Theorem 1.1. Now assume that $w \notin \cup_{p}\left(F^{\prime}\right)^{p} F^{\prime \prime}$. Then by Theorems 1.2 and 5.2, $w$ is a $\mathcal{N}$-word. We will argue as in the proof of Theorem 3.1.

Let $G$ be a finitely generated pronilpotent group, $d=d(G)$ and $H$ a free pronilpotent group on generators $x_{1}, \ldots, x_{d}, z$. Since $w$ is a $\mathcal{N}$-word, $\gamma_{n}\left(H^{t}\right) \leq \overline{w(H)}$ for some $n$ and $t$. We write $G$ as $G=G_{1} \times G_{2}$, where $G_{1}$ is the product of all the Sylow pro- $p$ subgroups with $p \in \pi(t)$ and $G_{2}$ is the product of the rest of the Sylow pro- $p$ subgroups. In the same way we write $H=H_{1} \times H_{2}$. Note that $w(G)$ is closed if and only $w\left(G_{1}\right)$ and $w\left(G_{2}\right)$ are closed. Since $w\left(G_{1}\right)$ is closed by Theorem 1.1, it is enough to prove only that $w\left(G_{2}\right)$ is closed. 
By our construction of $\mathrm{H}_{2}$ we have that $\gamma_{n}\left(H_{2}\right) \leq \overline{w\left(H_{2}\right)}$. Repeating the argument from the proof of Theorem 3.1, we obtain that any element of $\gamma_{n}\left(G_{2}\right)$ is a product of a bounded number of $w$-values in $G_{2}$. The StroudRoman'kov theorem $[20,15]$ says that any word $w$ has a finite width in a finitely generated nilpotent group. If the word $w$ has width at most $l$ in the free $d$-generator nilpotent group of class $n-1$, then $w$ has width at most $l$ in every finite quotient of this group; consequently $w\left(G_{2} / \gamma_{n}\left(G_{2}\right)\right)$ is closed. Thus we conclude that $w\left(G_{2}\right)$ is also closed.

\section{References}

[1] Barnea, Y. And Shalev, A.: Hausdorff dimension, pro- $p$ groups, and Kac-Moody algebras. Trans. Amer. Math. Soc. 349 (1997), 5073-5091.

[2] Bourbaki, N.: Lie groups and Lie algebras. Chapters 1-3. Elements of Mathematics (Berlin). Springer-Verlag, Berlin, 1989.

[3] Burns, R. G. And Medvedev, Yu.: Analytic relatively free pro- $p$ groups. J. Group Theory 7 (2004), 533-541.

[4] Dixon, J., du Sautoy, M., Mann, A. and Segal, D.: Analytic pro-p groups. Second edition. Cambridge Studies in Advanced Mathematics 61. Cambridge University Press, Cambridge, 1999.

[5] Govorov, V. E.: Graded algebras. Mat. Zametki 12 (1972), 197-204 (Russian). Translation in Math. Notes 12 (1972), 552-556.

[6] Hartley, B.: Subgroups of finite index in profinite groups. Math. Z. 168 (1979), 71-76.

[7] Huppert, B. and Blackburn, N.: Finite groups II. Fundamental Principles of Mathematical Sciences 242. Springer-Verlag, Berlin-New York, 1982

[8] Ivanov, S. V.: P. Hall's conjecture on the finiteness of verbal subgroups. Izv. Vyssh. Uchebn. Zaved. Mat. 325 (1989), 60-70. Translation in Soviet Math. (Iz. VUZ) 33 (1989), 59-70.

[9] Lubotzky, A. and Segal, D.: Subgroup growth. Progress in Mathematics 212. Birkhäuser Verlag, Basel, 2003.

[10] Merzluakov, Ju. I.: Verbal and marginal subgroups of linear groups. (Russian) Dokl. Akad. Nauk SSSR 177 (1967) 1008-1011.

[11] Neumann, H.: Varieties of groups. Springer-Verlag, New York, 1967.

[12] Newman, M. F., Schneider, C. and Shalev, A.: The entropy of graded algebras. J. Algebra 223 (2000), 85-100.

[13] Nikolov, N. And Segal, D.: On finitely generated profinite groups. I: strong completeness and uniform bounds. II: products in quasisimple groups. Ann. of Math. (2) 165 (2007), 171-238, 239-273. 
[14] Piontkovskir, D. I.: Hilbert series and relations in algebras. Izv. Ross. Akad. Nauk Ser. Mat. 64 (2000), 205-219 (Russian). Translation in Izv. Math. 64 (2000), 1297-1311.

[15] Roman'kov, V.A.: The width of verbal subgroups of solvable groups. Algebra i Logika 21 (1982), 60-72 (Russian). Translation in Algebra and Logic 21 (1982), 41-49.

[16] Segal, D.: Closed subgroups of profinite groups. Proc. London Math. Soc. (3) 81 (2000), 29-54.

[17] Segal, D.: Variations on a theme of Burns and Medvedev. Groups Geom. Dyn. 1 (2007), no. 4, 661-668.

[18] Serre, J. P.: Lie algebras and Lie groups. Second edition. Lecture Notes in Mathematics 1500. Springer-Verlag, 1992.

[19] Shalev, A.: Characterization of $p$-adic analytic groups in terms of wreath products. J. Algebra 145 (1992), 204-208.

[20] Stroud, P. W.: Topics in theory of verbal subgroups. PhD Thesis, University of Cambridge, 1966.

[21] Wilson, J. S.: Two-generator conditions for residually finite groups. Bull. London Math. Soc. 23 (1991), 239-248.

Recibido: 5 de marzo de 2007

Andrei Jaikin-Zapirain

Departamento de Matemáticas Facultad de Ciencias, módulo C-XV

Universidad Autónoma de Madrid

Campus de Cantoblanco, 28049 Madrid, Spain andrei.jaikin@uam.es

This work is supported by the joint project CAPES/MECD 065/04 of the Brazilian and Spanish Governments and by the Spanish Ministry of Science and Education, grant MTM2004-04665, partly with FEDER funds. 